\title{
An Improved Fusion Indoor Localization Method Using Bluetooth and Motion Sensor
}

\author{
Wenhao Fan ${ }^{1+}$, Qiuchen $\mathrm{Li}^{2}$, Kangjia Shao ${ }^{3}$, Ruoyu Chen ${ }^{4}$ and Zhiwei Lv ${ }^{5}$ \\ ${ }^{1}$ School of Software Engineering, University of Science and Technology of China
}

\begin{abstract}
In this paper, we describe an accurate and practical method about the localization of pedestrians in the indoor environment using Bluetooth technique. We introduce a whole system designed for overcoming the multipath effect of wireless signal to obtain the accurate location. Three major improvements have been made to the three most important procedures of localization algorithm. Our approach is designed for daily scenarios of multiple facilities, such as shopping mall and hospital. The proposed approach is tested on mobile devices, and the results of test show that the system is reliable and suitable for indoor localization in aiming scenarios. The error obtained is $0.336 \pm 0.102 \mathrm{~m}$, guaranteeing the accuracy of this method.
\end{abstract}

Keywords: bluetooth indoor localization, interpolation, iterative trilateration, particle filter.

\section{Introduction}

It has been a great interest in both localization academic community and industry to get location information and deliver well-planned services to aiming customers, especially in indoor environment. We need the indoor location of people because we want to use this location to do more benefits. For example, personal assistant should be more intimate and intelligent, such as turning on/off the light when you enter/leave one of your rooms, preparing your favourite suits when you finished your breakfast and then walked up to your closet. This kind of 'fantasy' should take place in every indoor circumstance in the near future.

The study about this kind of Location Based Service(LBS) emerged from the past days when smart phones had become a necessity but the technology and infrastructure were not perfect. The continually development of Internet of Things(IOT), $5^{\text {th }}$-Generation telecommunication, smart devices and fundamental networks, which are the solid footstone to LBS, makes the research about this field more promising.

Then the key problem becomes: how could we get the location of users? The ideal method must be reliable, highly accurate, cheap, and available. GPS is the most common positioning method. However, Line-of-Sight to four satellites makes it impossible in indoor scenario[1]. So the research of new technology is needed.

As we can see, most indoor localization methods with super high accuracy use the fusion of different kinds of wireless signals, such as Bluetooth, Wi-Fi, RFID and so on. But in most scenarios, signal fusion methods with multiple signals cost more, because of the earlier laying and maintenance. For techniques mentioned above, bluetooth has plenty of precious advantages, such as very accessible, ultra-low power consumption, easy laying and carrying, and economic. Other techniques have their problem in indoor environment. For Wi-Fi, the power consumption of Wi-Fi is too high to maintain a long-time positioning. And for RFID, it cannot communicate, which reduces the potential of LBS services. Other techniques like UWB and ultrasound are not available in most of our smart devices.

\footnotetext{
Corresponding author. Tel.: +86 18551582689
}

E-mail address: sa615165@mail.ustc.edu.cn. 
A lot of efforts have been made in indoor localization used bluetooth. [2] utilized angle of arrival for localization, which required an antenna array to measure the angle of signals, resulted in an expensive and customized system. [3] proved that bluetooth performed well in indoor localization. [4] used RSSI(Received Signal Strength Indication) as an indicator of RX power level and achieved mean error of $3.5 \mathrm{~m}$. [5][6] used RSSI achieved 2.06 m and 1.52-3.0 m mean error. Methods mentioned above considered this problem as a static positioning, however, neglecting the motion is not appropriate and motions can also help us to correct the positioning. [7] proposed a method which can be used in tracking pedestrians with mean error $1.67 \mathrm{~m}$. But they only considered the corridors, hence this system performs badly in big rooms like lobbies.

Therefore, we intend to achieve an indoor positioning system which could obtain users' location despite moving or staying still, with the accuracy $<0.5 \mathrm{~m}$ and based only on bluetooth and users' smart devices. Meanwhile, the system should fit multiple indoor environment. In this paper, we proposed a method achieved $0.323 \pm 0.102 \mathrm{~m}$ in static and $0.355 \pm 0.236 \mathrm{~m}$ in mobile, which is better in both performance and system design.

The paper is organized as follows: Section 2 summarizes the overview and defects of RSSI-based bluetooth positioning and tracking techniques. Section 3 introduces our improvement to the positioning part of the whole localization system. Section 4 presents the tracking method we developed with particle filter and Kalman filter.

\section{RSSI-Based Bluetooth Localization and Challenge}

\subsection{Bluetooth localization technique}

Typical indoor localization method with bluetooth requests building a mapping model between distance and RSSI. This method includes two steps, first we measure the RSSI value of every anchor point(AP) received from user's device, then use LSE (least squares method) and trilateration or fingerprinting method to estimate the location. But due to the multipath effect, results from the procedure above are very unreliable.

\subsection{RSSI-Distance Mapping}

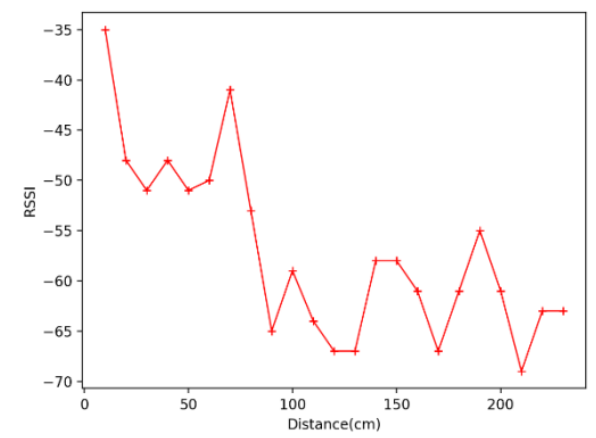

Fig. 1: RSSI changes with the distance.

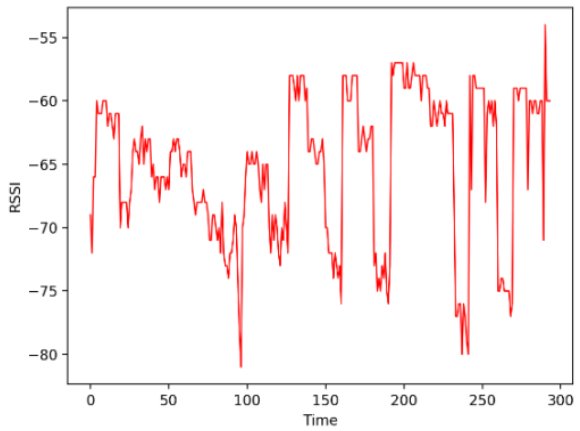

Fig. 2: Variance of RSSI over time at $3 \mathrm{~m}$ distance. 
In free environment, the RX power of wireless signal reduces with distance. And the distance and RSSI are supposed to obey the logarithmic normal distribution(LND). But this mapping influenced by physical space and overlay of the wireless signal, which is also known as shadowing and multipath effect, respectively. Hence if making the distance between two bluetooth devices changing steadily, the RSSI readings will not be stable, like figure 1 . In the meantime, the mapping of distance and RSSI reading will not perfectly fit the LND, like figure 2 .

[8] analyzed three methods of mapping the distance to RSSI. One of the method is interpolation, this method uses two reference nodes to realize a more accurate estimate of distance. First we use the radio wave propagation model. (1), (2) are the equations fitted for two reference nodes. $P_{T X}$ and $P_{R X}$ are the transmitting and receiving power level measured by the mobile devices. $G_{T X}$ and $G_{R X}$ are antenna gains of the transmitter and the receiver. $\lambda$ is wavelength and $n$ denotes the influence of obstacles. $X_{\alpha}$ is a normal random variable representing error. $d_{1}$ and $d_{2}$ are two arbitrary reference distances. The interpolation model can be derived by $\left(P_{R X}-P_{R X 2}\right) /\left(P_{R X 1}-P_{R X 2}\right)$ as (3). $\mathrm{d}$ is the distance we got from this method and the value is trustworthy.

$$
\begin{gathered}
P_{R X 1}=P_{T X}+G_{T X}+G_{R X}+20 \log (\lambda)-20 \log (4 \pi)-10 n \log \left(\mathrm{d}_{1}\right)-X_{\alpha} \\
P_{R X 2}=P_{T X}+G_{T X}+G_{R X}+20 \log (\lambda)-20 \log (4 \pi)-10 n \log \left(\mathrm{d}_{2}\right)-X_{\alpha} \\
P_{P X}=\left(P_{R X 1}-P_{R X 2}\right) \frac{\log (\mathrm{d})-\log \left(\mathrm{d}_{2}\right)}{\log \left(\mathrm{d}_{1}\right)-\log \left(\mathrm{d}_{2}\right)}+P_{R X 2}
\end{gathered}
$$

And reference points work every time instead of using them just for training readings of APs. After this modification, the instability of RSSI is eliminated in real time, most influence from the environment is compensated, so the result contains information more from real signal and less from shadowing or multipath. This improvement only adds a little calculation, so it won't be a burden to mobile system.

\section{Estimation of Location}

After getting the distance from devices to AP, next step is to ensure the location. Methods mentioned commonly are fingerprinting and trilateration. Fingerprinting contains offline and online phase. In the offline phase, the room is divided into areas. Signals are collected to build characters of each area. In the online phase, system compares the characters in real time with the characters built before. The more similar the characters are, the more likely they are in the same area. But fingerprinting needs preliminary manpower to get characters, especially when the room is huge or changes frequently. Another problem is that the calculation of comparison is too heavy for mobile devices. And the accuracy of fingerprinting relies on the granularity of division.

The main idea of trilateration is to calculate the location based on the distance of APs, which the location of APs are already known. Usually we use three AP locations and target distances from APs to draw three circles, and the intersect should be the location. But interference may jeopardize the received reading. So the calculation aimed at minimizing errors may be the best way to estimate the position.

\subsection{Classic Approach}

For calculating the result with minimum error of distance-coordinates pairs, the first thought is to take every pair into account. Supposing that we have $\mathbf{n}$ pairs of distance and AP location, using $d$ as the distance, $(x, y)$ and $\left(x_{i}, y_{i}\right)$ as the coordinates of target device and AP respectively, then we have (3). Using every equation minus the last equation, and transforming them to matrix form, then we have (4).

After we estimating the standard minimum mean squared error model, the location of target device is $X=\left(A^{T} A^{-1}\right) A^{T} b$. This method is easy to implement, but is not reliable because of using the absolute error.

$$
\begin{gathered}
\left\{\begin{array}{l}
\left(x_{1}-x\right)^{2}+\left(y_{1}-y\right)^{2}=d_{1}^{2} \\
\cdots \\
\left(x_{n}-x\right)^{2}+\left(y_{n}-y\right)^{2}=d_{n}^{2}
\end{array}\right. \\
X=\left(\begin{array}{ll}
x \quad y
\end{array}\right)^{T}, A=\left(\begin{array}{cc}
2\left(x_{1}-x_{n}\right) & 2\left(y_{1}-y_{n}\right) \\
\vdots & \vdots \\
2\left(x_{n-1}-x_{n}\right) & 2\left(\mathrm{y}_{n-1}-y_{n}\right)
\end{array}\right), b=\left(\begin{array}{c}
x_{1}^{2}-x_{n}^{2}+y_{1}^{2}-y_{n}^{2}+d_{1}^{2}-d_{n}^{2} \\
\vdots \\
x_{n-1}^{2}-x_{n}^{2}+y_{n-1}^{2}-y_{n}^{2}+d_{n-1}^{2}-d_{n}^{2}
\end{array}\right)
\end{gathered}
$$




\subsection{Iterative Approach}

As our target is to find the minimum error result which fits our data measured from APs, minimizing error becomes an optimization problem. Then we introduce gradient descent, a traditional method for optimization problem, into this problem, and [9] also used this idea. The loss function represents the error we get, in this problem, the loss function is:

$$
\left|f_{i}\right|=\left|d_{i}-\sqrt{\left(x_{i}-x_{e}\right)^{2}+\left(y_{i}-y_{e}\right)^{2}}\right|
$$

$f$ represents the error of our result, $d$ represents the distance we measured, $\left(x_{i}, y_{i}\right)$ and $\left(x_{e}, y_{e}\right)$ represents the coordinates of AP number $i$ and estimates target location respectively. As we can see from the section before, the distance-RSSI mapping obeys LND. When RSSI is small, it represents that the AP is far from target device, and a little wave of RSSI will cause the estimated distance changing dramatically, so the reliance of RSSI data should be low when the RSSI reading is small, so as the weight of corresponding RSSI reading. But in (5), the weight of small RSSI readings is high because the distance may be large, so we make some modification as equation (6), and through this way we make the weights closer to real situation:

$$
\left|f_{i}\right|=\left|\frac{d_{i}-\sqrt{\left(x_{i}-x_{e}\right)^{2}+\left(y_{i}-y_{e}\right)^{2}}}{d_{i}}\right|
$$

Then the adjustments $(\Delta x, \Delta y)$ used in every iteration should be like (7), in matrix form:

$$
\begin{gathered}
\Delta=\left(B^{T} B\right)^{-1} B^{T} f \text { or } \Delta=(\Delta x, \Delta y)^{T} \\
B=\left(\begin{array}{cc}
\partial f_{1} / \partial x_{e} & \partial f_{1} / \partial y_{e} \\
\vdots & \vdots \\
\partial f_{i} / \partial x_{e} & \partial f_{i} / \partial y_{e}
\end{array}\right)=\left(\begin{array}{cc}
\frac{\left(x_{1}-x_{e}\right)}{\sqrt{\left(x_{1}-x_{e}\right)^{2}+\left(\mathrm{y}_{1}-y_{e}\right)^{2}}} & \frac{\left(\mathrm{y}_{1}-y_{e}\right)}{\sqrt{\left(x_{1}-x_{e}\right)^{2}+\left(\mathrm{y}_{1}-y_{e}\right)^{2}}} \\
\vdots & \vdots \\
\frac{\left(x_{i}-x_{e}\right)}{\sqrt{\left(x_{i}-x_{e}\right)^{2}+\left(\mathrm{y}_{i}-y_{e}\right)^{2}}} & \frac{\left(\mathrm{y}_{i}-x_{e}\right)}{\sqrt{\left(x_{i}-x_{e}\right)^{2}+\left(\mathrm{y}_{i}-y_{e}\right)^{2}}}
\end{array}\right) \\
x_{e}=x_{e}+k \Delta x \text { and } y_{e}=y_{e}+k \Delta y
\end{gathered}
$$

The matrix $B$ in (7) is shown in (8), and the update equation is (9). $k$ is the parameter we could use to adjust the speed of convergence.

\section{Localization Filter}

For everything we discussed above, only the physical space was used, but the temporal factor would also help in localization. Using former time location to estimate the present location would be a great idea. So we introduce Kalman filter and particle filter to take the former state and the measurement of the system all into account, and we will decide how much we trust in these two parts. To make our system more practical, we use virtual step counter sensor to distinguish the situation in order to use different filter.

\subsection{Particle Filter}

The main object of particle filter is to track the state of a target variable as it transforms over time. [10] is about to build probability distribution function in a form of sampling. Multiple particles are used to represent the different possibilities of system state. The weight of particle shows the significance to system state. Particle filter algorithm have three step: prediction, update and resample. We continually do these three steps after every action of system to obtain more accurate estimate of the target location.

Prediction procedure: According to the model we designed, we make a new estimate of the state of particles in every action. Update procedure: Based on the measurement of sensor or bluetooth device, we calculate the weights of every particle. After serval actions, the weight of particles polarizes: the weight of most particles comes to zero, only few particles still weight heavily. This effect is degeneracy and it costs most of power used in the calculation of near-zero particles. Resample means to eliminate the ones with small weights and duplicate the particles with heavy weights.

But the resample may cause the sample impoverished. Then we use the coefficient of variation $c v_{t}^{2}$ and effective sample size $E S S_{t}$. And we do resample only when the $E S S_{t}$ value reduces to a certain threshold. 


$$
\begin{gathered}
c v_{t}^{2}=\frac{\operatorname{var}\left(\omega_{t}(i)\right)}{E^{2}\left(\omega_{t}(i)\right)}=\frac{1}{M} \sum_{i=1}^{M}(M \omega(i)-1)^{2} \\
E S S_{t}=M / 1+c v_{t}^{2}
\end{gathered}
$$

\subsection{Kalman Filter}

Half of the circumstance of indoor localization is without moving, which would be a huge waste of power to use particle filter all time. In this situation, the biggest problem is that the location calculated from trilateration wanders unpredictable. But Kalman filter perfectly fits these situations because it calculates the Kalman gain to balance the data from past and measurement.

We use virtual step counter senor to evaluate the moving status of pedestrians. This virtual sensor is common in smart phones and wrist bands. It uses accelerometer and magnetometer to detect the pattern of people's movement to count steps. When pedestrians move very slowly, Kalman filter is easy and energy-saving. But particle filter is taken back online when pedestrians are walking or in other high speed movement.

\section{Experiments}

A $16^{*} 14$ room is used for experiments as figure 3, and there are several long tables and pillars in this room. Bluetooth APs are planted as a matrix and the distance between each other is $3 \mathrm{~m}$, showing as red round.

\subsection{Signal Character}

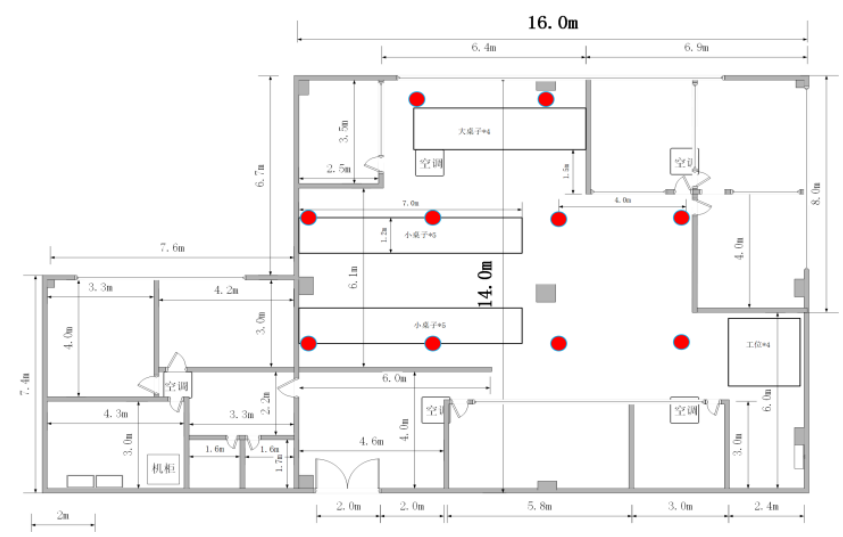

Fig. 3: Test room and layout of bluetooth APs.

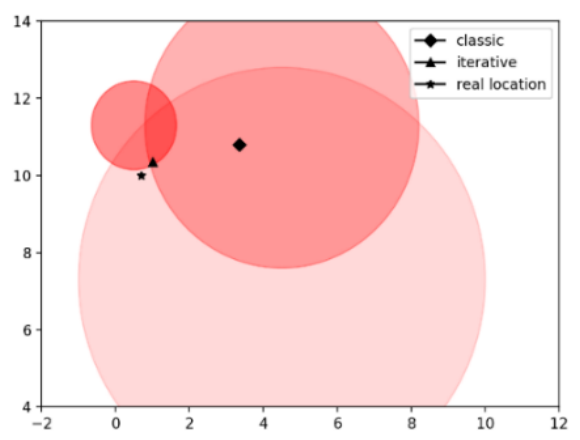

Fig. 4: Full-batch approach vs iterative approach.

We already showed the signal characters in figure 1, which RSSI is highly unstable with time at a fixed distance. And the mapping between distance and RSSI fits LND but influenced from the problem of noise. So the introduction of interpolation used to compensate the noise is necessary. Using interpolation and iterative trilateration, we achieved an accurate estimate of target device. As we can see, in the example of 
figure 4, the iterative approach performs much better than classic one. In this example, the locations of three APs are $(0.5,11.3),(4.5,11.3),(4.5,7.3)$, and corresponding estimate distances are 1.15, 3.702, 5.5. Target location is $(0.7,10)$. The result of iterative approach is $(1,10.35)$ while the result of classic approach is $(3.345,10.8)$

\subsection{Static Localization}

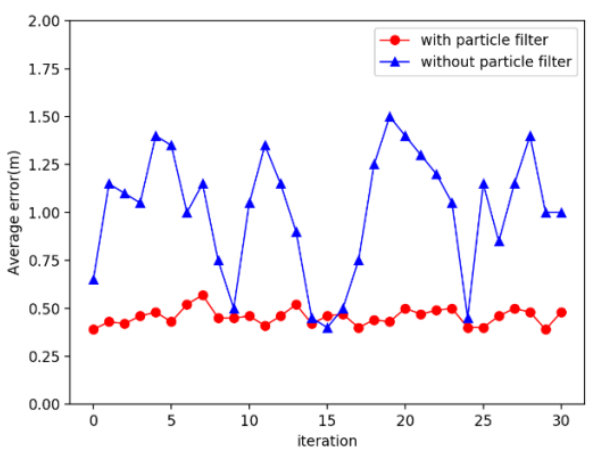

Fig. 5: Comparison of error with or without kalman filter in static localization scenario.

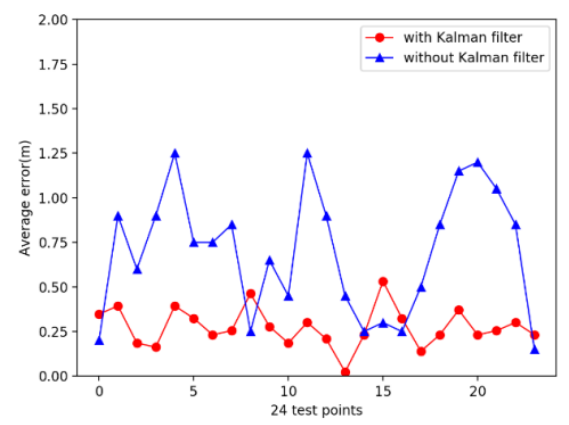

Fig. 6: Comparison of error with or without particle filter in mobile localization scenario.

Figure 5 shows the comparison of error over 24 test points. The average error after using Kalman filter is compared with the iterative approach error, including locations near the wall or pillar. This graph shows system used Kalman filter to eliminate the error after the iterative trilateration and makes the average error $<0.5 \mathrm{~m}$.

\subsection{Mobile Localization}

Figure 6 shows the comparison of error over 30 iterations of mobile localization, which means we track the target over 30 actions. The system error stays very low at whole time, which means the particle filter successfully used the former location and measurement to estimate the current location. And system shows robustness over long time and the occasionally large error.

\subsection{Overall Results}

Table 1: Error in different scenarios

\begin{tabular}{|c|c|l|}
\hline No. of scenario & Error & Comments \\
\hline 1 & $0.323 \pm 0.102$ & No evident barrier to signal in static scenario \\
\hline 2 & $0.536 \pm 0.299$ & Near pillar or table in static scenario \\
\hline 3 & $0.355 \pm 0.236$ & Free walking \\
\hline 4 & $0.482 \pm 0.167$ & Walking through tables and pillars \\
\hline
\end{tabular}


The results of various scenarios are shown in Table 1 . The mean error of static localization is $0.323 \mathrm{~m}$ with standard deviation of $0.102 \mathrm{~m}$ in circumstance with no evident barriers. The positioning in environment with obstacles is always a difficulty, but the proposed system performs reasonable in room with low density obstacles. The error is $0.536 \mathrm{~m}$ with standard deviation of $0.299 \mathrm{~m}$ in this situation, still acceptable. So does the performance in mobile localization, $0.355 \pm 0.236 \mathrm{~m}$ was achieved in free walking with no evident obstacles and $0.482 \pm 0.167 \mathrm{~m}$ was achieved in room with low density obstacles shows in figure 4 .

\section{Conclusion and Future Work}

In this paper, we proposed a positioning system which can be used in indoor environment with accuracy less than $0.5 \mathrm{~m}$. The algorithm of this system is based on bluetooth and step sensor, which is easy to implement on mobile devices. We used step counter to divide the motion pattern into static and mobile in order to use different filters to cut the power consumption. We achieved $0.323 \pm 0.102 \mathrm{~m}$ in static localization and $0.355 \pm 0.236 \mathrm{~m}$ in mobile. And the error in the situation with evident obstacles is less than $0.6 \mathrm{~m}$ in both static and mobile. In the future, we plan to use the channel information to achieve localization method with higher accuracy and stability.

\section{Acknowledgements}

We acknowledge A.P Yang Wang form University of Science and Technology of China for unselfishly offering many help in the researching process of this paper.

\section{References}

[1] A. Harder, L. Song and Y. Wang, Towards an indoor location system using RF signal strength in IEEE 802.11 networks, Proc. Int. Conf. on IT: Coding and Computing, Apr. 2005, pp 228-233.

[2] A. Kotanen, M. Hannikainen, H. Leppakoski and T. D. Hamalainen, Experiments on Local Positioning with Bluetooth, Proc. Int. Conf. on IT: Coding and Computing, 2003, pp 297-303.

[3] J. Hallberg, M. Nilsson, and K. Synnes. Positioning with bluetooth. ICT 2003: 10th International Conference on Telecommunications, volume 2, Feb- Mar 2003, pp 954-958 vol.2.

[4] Kotanen A., Hannikainen M., Leppakoski H. and Hamalainen T.D, Experiments on local positioning with Bluetooth. ITCC 2003: International Conference on Information Technology: Coding and Computing, 2003. Proceedings, 28-30 Apr. 2003, pp 297-303.

[5] Silke Feldmann, Kyandoghere Kyamakya, Ana Zapater. An indoor bluetooth-based positioning system: Concept, implementation and experimental evaluation. International Conference on Wireless Networks, 2003, pp 109-113.

[6] Varun Almaula and David Cheng, Bluetooth Triangulator, Final Project, Department of Computer Science and Engineering, University of California, San Diego, 2006

[7] Au, Anthea Wain Sy, et al. Indoor tracking and navigation using received signal strength and compressive sensing on a mobile device. IEEE Transactions on Mobile Computing Dec. 2013, pp 2050-2062.

[8] Bing-Fei Wu, Cheng-Lung Jen, and Kuei-Chung Chang. Neural fuzzy based indoor localization by Kalman filtering with propagation channel modeling. IEEE International Conference on Systems, Man and Cybernetics, Oct. 2007, pp 812-817.

[9] Erin-Ee-Lin Lau and Wan-Young Chung. Enhanced RSSI-based real-time user location tracking system for indoor and outdoor environments. ICCIT 07: Proceedings of the 2007 International Conference on Convergence Information Technology, 2007, pp 1213-1218.

[10] Ioannis M. Rekleitis, A particle filter tutorial for mobile robot localization, Technical Report TR-CIM-04-02, Centre for Intelligent Machines, McGill University, Apr 2004.

[11]Liu, Jun S., Rong Chen, and Tanya Logvinenko. A theoretical framework for sequential importance sampling with resampling. Sequential Monte Carlo methods in practice. Springer, New York, NY, 2001, pp 225-246. 